\title{
Outcomes following total laryngectomy for squamous cell carcinoma: One centre experience
}

\author{
S.C. Leong ${ }^{a}$, S.-S. Kartha ${ }^{a}$, C. Kathan ${ }^{b}$, J. Sharp ${ }^{a}$, S. Mortimore ${ }^{a, *}$ \\ a Department of Otolaryngology - Head \& Neck Surgery, Derby Royal Infirmary, Derby DE1 2QY, United Kingdom \\ ${ }^{\mathrm{b}}$ Faculty of Economics, Administrative and Social Sciences, Bilkent University, 06800 Ankara, Turkey
}

\section{KEYWORDS \\ Laryngectomy; \\ Outcome; \\ Cancer; \\ Larynx; \\ Hypopharynx}

\begin{abstract}
Summary
Objectives: To evaluate the clinical outcomes of total laryngectomy (TL), complications and factors affecting survival.

Design: Retrospective review of hospital electronic database for head and neck squamous cell carcinoma (SCCa).

Setting: Large district general hospital in England, United Kingdom.

Participants: Patients who had TL between January 1994 and January 2008.

Main outcome measures: 5-year disease specific survival (DSS) and disease-free survival (DFS). Results and conclusions: Seventy-one patients were reviewed, of whom 38 (54\%) had laryngeal SCCa and 33 (46\%) hypopharyngeal SCCa. The overall mean survival period following TL was 42.4 months. The 5-year DSS and DFS was better for laryngeal SCCa compared to hypopharyngeal SCCa, although not statistically significant $(P=0.090, P=0.54$ respectively). Patients treated for laryngeal SCCa had a mean survival period of 47.5 months compared to 36.5 months for hypopharyngeal disease. Those who had laryngeal recurrence after primary radiotherapy (RT) demonstrated statistically better survival probability than those who had hypopharyngeal recurrence $(P=0.011)$. Patients without cervical lymphadenopathy had statistically better survival $(P=0.049)$. The most common early complication was related to the cardiorespiratory system. One fatal complication of erosion of the brachiocephalic artery due to the laryngectomy tube was noted. The most common late complication was neopharyngeal stenosis. The commonest cause of death was due to locoregional recurrence, followed by medical co-morbidities. Patients referred to specialised head and neck clinic had a better survival probability than those referred to a general ENT clinic $(P=0.37)$. While there is increasing tendency towards laryngeal conservation, total laryngectomy remains a robust treatment option in selected patients. (c) 2012 Published by Elsevier Masson SAS.
\end{abstract}

\section{Introduction}

Laryngeal cancer is the commonest carcinoma of the head and neck region [1]. In 2005, 2190 patients were diagnosed in the United Kingdom and caused 800 deaths in 2006 [2]. Despite advances in chemo-radiotherapy (RT), surgery

\footnotetext{
* Corresponding author.

E-mail address: sean.mortimore@derbyhospitals.nhs.uk
} (S. Mortimore).

1879-7296/\$ - see front matter @ 2012 Published by Elsevier Masson SAS. 
continues to play an important role in the management of laryngeal cancer. Laryngeal preservation techniques such as endoscopic laser resection have been increasing popular. However, total laryngectomy (TL) remains a reasonable option for advanced disease in selected patients.

Whilst there are numerous studies comparing outcomes of different treatment arms for laryngeal carcinoma, there are scarce data on patients who have undergone $T L$, especially with regards to long-term outcome and prognosis. Outcome data would improve our ability to council these patients regarding important therapeutic decisions and end-of-life issues. The aim of this paper was to retrospectively evaluate the clinical outcomes of $T L$, postoperative complications and factors affecting survival rate. The impact of surgery on quality of life (QoL) was not included in this review as not all patients had QoL evaluation, especially those who had surgery at the beginning of the review period. Furthermore, it was not possible to retrospectively evaluate QoL issues as some of the patients had died.

\section{Methods}

\section{Patients}

The ENT Department at Derby Hospitals NHS Foundation Trust is part of the regional head and neck cancer network covering southern Derbyshire and eastern Staffordshire (England, UK), serving a population of over 800,000 . A retrospective review of the departmental electronic cancer database was undertaken between January 1994 and January 2008. Patients who had TL for squamous cell carcinoma (SCCa) of the larynx and hypopharynx were included, as were those who had TL as a salvage procedure for recurrence following either primary $\mathrm{RT}$ or transoral $\mathrm{CO}_{2}$ laser resection (TOLR). Recurrence was defined as histological evidence of lesion at least 12 months after completion of the primary treatment. Patients with primary cervical oesophageal cancers and those who had less than 6 months follow-up were excluded. The departmental database and patient case notes were reviewed for epidemiological data, tumour stage, complications and survival outcomes.

All patients were staged according to the International Union Against Cancer (UICC, 2002)/American Joint Commission on Cancer (AJCC, 2002) staging system. Preoperative staging of tumour was performed by endoscopy and radiological imaging (CT neck and chest). The management of all patients was discussed at the head and neck multidisciplinary team (MDT) meeting. Patients were counselled regarding possible treatment options and informed consent for TL was obtained. All patients received a primary tracheooesophageal puncture, which was used for feeding until oral intake was established. Therapeutic neck dissection was performed at the time of laryngectomy in patients with cervical node involvement. All operations were performed by either JS or SM, who were the senior surgeons in the department. Postoperative RT was given to the primary site and neck based on clinicopathological risk factors including status of resection margins, perineural invasion, lymph node involvement and the presence of extracapsular nodal spread.
Complications were categorised as early and late onset. Early complications were defined as those arising within four weeks of surgery and late complications were those occurring subsequently. Where patients had died in the community, their General Practitioner (GP) was contacted to obtain the date and cause of death. The standard followup regime in our institution following TL is monthly review in the 1st year, 2-monthly in the 2nd year, 4-monthly in the 3 rd and 4th year and 6-monthly in the 5th year. Patients are normally discharged from follow-up after 5 years but were advised to return if they had any concerns. In addition, all patients had access to the Clinical Nurse Specialist in Head and Neck Cancer, including those who had been discharged.

\section{Ethical considerations}

Institutional Review Board approval was not required for this retrospective review. The encrypted electronic database was kept on a hospital computer in a secured location, accessed only by password.

\section{Statistical analysis}

Statistical analysis of the data was performed with SPSS 14.0 computer software (SPSS Inc., IL, USA). All survival probabilities were estimated by using the Kaplan-Meier method from the day of TL. Log-rank tests (Cox Mantel) were performed to compare differences between the estimates. Results were regarded as statistically significant if $P \leq 0.05$. The cumulative 5-year disease specific survival (DSS) and disease-free survival (DFS) probabilities were evaluated. Mean survival period, together with standard error (SE) and 95\% confidence interval $(95 \% \mathrm{Cl})$ were also calculated.

\section{Results}

The review period spanned 14 years. Seventy-one patients (61 males, 10 females) fulfilled the inclusion criteria for this study. The mean age of patients was 64 years (range 40-84 years). The postoperative follow-up period ranged from 6 months to 128 months (mean 42 months). Patients stayed in hospital for an average of 16 days (range 12-20) after surgery.

Thirty-eight (54\%) patients were diagnosed with laryngeal SCCa and 33 (46\%) had hypopharyngeal SCCa. Most of the patients (83\%) presented with either T3 or T4 SCCa (Table 1) and had Stage IV disease. The remaining $17 \%$ had T1 or T2 tumours at presentation, all of whom had previously undergone either RT or TOLR. Of the 54 patients who had surgery as the primary treatment modality, 46 (65\%) had TL and eight (11\%) total laryngo-pharyngectomy (two jejunal free-flap, six gastric pull-up reconstruction procedure). The remaining $17(24 \%)$ patients had salvage surgery, of whom $16(23 \%)$ had previously been treated with RT and one patient had TOLR. Of these, 15 patients had TL and two had total laryngopharyngectomy and jejunal free-flap reconstruction. Nearly half of the cohort (48\%) had neck dissection procedures at the time of laryngeal resection.

Although there were no intra-operative complications, 24 early postoperative complications were recorded (Table 2 ). 
Table 1 Clinical stages of laryngeal cancer $(n=38)$ and hypopharyngeal cancer $(n=33)$.

\begin{tabular}{|c|c|c|c|c|c|c|c|c|c|c|c|}
\hline \multirow[t]{2}{*}{ Larynx $(n=38)$} & \multicolumn{4}{|c|}{ Untreated } & \multicolumn{7}{|c|}{ Previously treated } \\
\hline & $\mathrm{T} 2$ & T3 & $\mathrm{T} 4$ & Total & $\mathrm{T} 1$ & $\mathrm{~T} 2$ & T3 & & T4 & & Total \\
\hline NO & 3 & 2 & 9 & 14 & 4 & $2^{a}$ & 4 & & 1 & & 10 \\
\hline N1 & & 1 & 3 & 4 & & 1 & & & & & 1 \\
\hline N2 & & 1 & 5 & 6 & & & 1 & & & & 1 \\
\hline N3 & & & 1 & 1 & & & & & & & \\
\hline Total & 3 & 4 & 18 & 25 & 4 & 2 & 5 & & 1 & & 13 \\
\hline \multirow[t]{2}{*}{ Hypopharynx $(n=33)$} & & \multicolumn{4}{|c|}{ Untreated } & \multicolumn{6}{|c|}{ Previously treated } \\
\hline & & $\mathrm{T} 2$ & $\mathrm{~T} 3$ & $\mathrm{~T} 4$ & Total & $\mathrm{T}$ & & $\mathrm{T} 4$ & & Total & \\
\hline NO & & & 2 & 11 & 13 & & & & & & \\
\hline N1 & & 1 & 1 & 3 & 5 & 1 & & 2 & & 3 & \\
\hline N2 & & & 1 & 7 & 8 & & & 2 & & 2 & \\
\hline N3 & & & & 2 & 2 & & & & & & \\
\hline Total & & 1 & 4 & 23 & 28 & 1 & & 4 & & 5 & \\
\hline
\end{tabular}

a Includes one patient previously treated with transoral $\mathrm{CO}_{2}$ laser resection.

Table 2 Summary of early and late complications.

\begin{tabular}{ll}
\hline Description & Number of cases (\%) \\
\hline Early complication & $4(17)$ \\
Pneumonia & $4(17)$ \\
Cardiac arrhythmias & $4(17)$ \\
Hypocalcemia & $3(13)$ \\
Pharyngo-cutaneous fistula & $2(8)$ \\
Wound haematoma & $2(8)$ \\
Deep vein thrombosis & $2(8)$ \\
Wound infection & $2(8)$ \\
Chyle leak & $1(4)$ \\
Erosion of brachiocephalic artery & $24(100)$ \\
Total & \\
Late complications & $4(29)$ \\
Neopharyngeal stenosis & $3(21)$ \\
Tracheo-stomal stenosis & $3(21)$ \\
Tracheo-oesophageal puncture & \\
related & $1(6)$ \\
Neuroma of the neck & $1(6)$ \\
Oesophageal stricture & $1(6)$ \\
Pharyngeal leak & $1(6)$ \\
Hypothyroidism & $1(6)$ \\
Jejunal anastomotic stenosis & $14(100)$ \\
Total &
\end{tabular}

The most common was related to the cardiorespiratory system (34\%), frequently associated with pre-existing medical co-morbidities. Transient hypocalcaemia which resolved prior to hospital discharge was recorded in four cases. All cases of chyle leak and pharyngo-cutaneous fistula (PCF) were managed conservatively. Of the three cases with PCF, only one patient had primary RT prior to TL. The two cases of wound infection, managed conservatively with antibiotics, did not result in wound dehiscence. One fatal complication of erosion of the brachiocephalic artery due to the laryngectomy tube was noted. The most common late complication was neopharyngeal stenosis $(29 \%)$, followed by trachea-stomal stenosis $(21 \%)$ and problems with the tracheo-oesophageal puncture site (Table 2). Both cases of oesophageal and jejunal anastomotic stenosis were dilated endoscopically. There was one case of hypothyroidism, which required long-term thyroxine replacement.

Twenty-six patients (36.6\%) remained alive during the review period (Fig. 1), of whom 11 had been treated for laryngeal SCCa and 15 for hypopharygeal SCCa. The commonest cause of death was due to locoregional recurrence (36\%), followed by medical co-morbidities (Table 3 ). There were eight $(11.3 \%)$ recorded cases of distant metastasis, the most common being to the lung. Six patients $(8.5 \%)$ had a second primary cancer diagnosed. The average time interval to diagnosis of the second primary was 17.6 months (range 3-55). The overall mean survival period following TL was 42.4 months (SE 2.8, 95\% Cl 37.0-47.9). Survival outcome was not statistically significant between the male

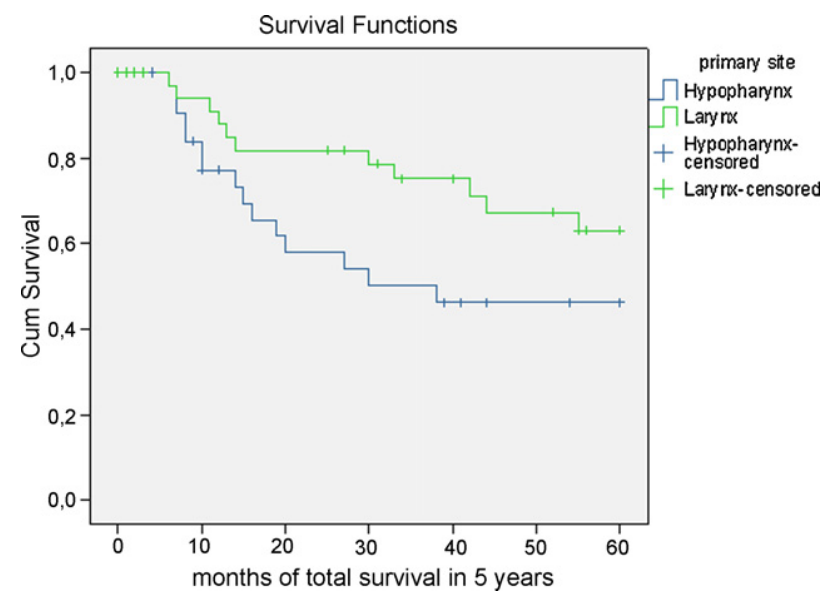

Figure 1 Laryngeal and hypopharyngeal squamous cell carcinoma, 5-year disease specific survival (DSS) compared. NS $(P=0.090)$. 
Table 3 Cause of death following total laryngectomy.

\begin{tabular}{ll}
\hline Cause of death & Number of deaths (\%) \\
\hline Erosion of brachiocephalic artery & $1(2)$ \\
Locoregional recurrence & $16(36)$ \\
Distant metastasis & \\
Lung & $6(14)$ \\
Liver & $1(2)$ \\
Bone & $1(2)$ \\
Second primary carcinoma & \\
Bronchogenic & $2(4)$ \\
Oesophagus & $2(4)$ \\
Tonsil & $1(2)$ \\
Tongue & $1(2)$ \\
Medical co-morbidities & \\
Cardiovascular disease & $6(14)$ \\
End-stage respiratory disease & $3(7)$ \\
Bronchopneumonia & $3(7)$ \\
Acute renal failure & $1(2)$ \\
Septicaemia & $1(2)$ \\
Total & $45(100)$ \\
\hline
\end{tabular}

and female cohorts $(P=0.75)$. The overall DSS and DFS was 0.39 and 0.54 respectively at 5 years. Although the 5 year DSS (Fig. 2) and DFS (Fig. 3) was better for laryngeal SCCa compared to hypopharyngeal SCCa, the difference was not statistically significant $(P=0.090, P=0.54$ respectively). Patients treated for laryngeal SCCa had a mean survival period of 47.5 months (SE 3.4, 95\% Cl 40.9-54.2) compared to 36.5 months (SE 4.3, 95\% Cl 28.0-45.0) for hypopharyngeal disease.

Those who had laryngeal recurrence after primary RT demonstrated statistically better survival probability than those who had hypopharyngeal recurrence $(P=0.011$, Fig. 3). These patients survived a mean of 53.2 months (SE 4.7, $95 \% \mathrm{Cl} 44.1-62.4$ ) compared to 19.6 months (SE 6.0, $95 \% \mathrm{Cl} 7.9-31.3)$ in those with recurrent hypopharyngeal

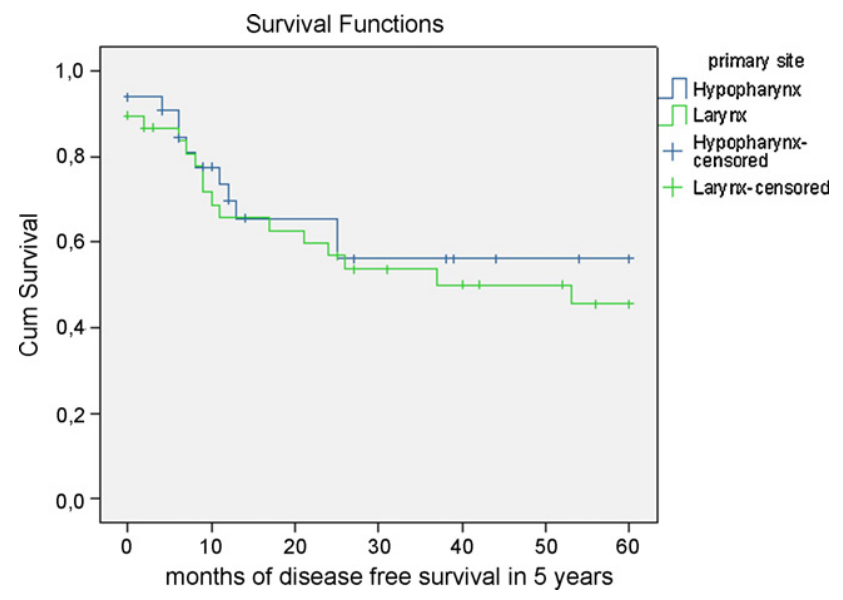

Figure 2 Laryngeal and hypopharyngeal squamous cell carcinoma 5-year disease-free survival (DFS) rates compared. NS $(P=0.54)$.

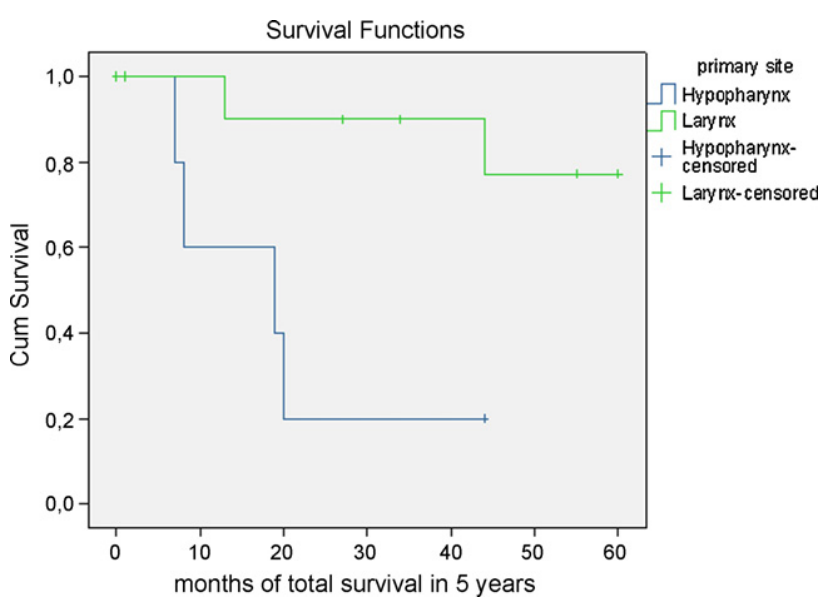

Figure 3 Previously treated laryngeal and hypopharyngeal squamous cell carcinoma 5-year disease specific survival (DSS) rates compared $(P=0.011)$.

disease. The mean period to recurrence after completion of primary RT was 12.9 months (range 0-53) for laryngeal SCCa and 14.5 months (range 0-72) for hypopharyngeal SCCa. Patients who had TL as the primary treatment modality for laryngeal SCCa demonstrated a non-significant trend towards poorer survival (mean 44.5 months, SE $4.5,95 \% \mathrm{CI}$ 35.7-53.2) than primary RT. However, patients who had primary TL for hypopharyngeal SCCa had better survival probability $(P=0.127)$ compared to those who had primary RT. Those who had surgery survived a mean of 39.5 months (SE 4.7, 95\% Cl 30.2-48.7) compared to 19.6 months (SE 6.0, 95\% Cl 28.0-45.0) in patients who had primary RT. Of the 54 patients who had TL as the primary treatment modality, $83 \%$ were Stage IV disease at presentation. The mean survival period for Stage IV laryngeal SCCa was 44.8 months (SE 4.6, 95\% Cl 35.8-53.8), compared to 36.2 months (SE 4.7, 95\% Cl 26.9-45.4) for Stage IV hypopharyngeal SCCa $(P=0.333)$.

Laryngeal SCCa patients who were NO at presentation had statistically better survival $(P=0.049)$, averaging 51.9 months (SE 3.9, $95 \% \mathrm{Cl} 44.7-59.1$ ), compared to those who were $\mathrm{N}$ positive (mean 38.7 months, SE $6.2,95 \% \mathrm{Cl}$ 26.5-50.9). This trend was similarly observed in hypopharyngeal SCCa, although it did not achieve statistically significance $(P=0.122)$. Those without cervical involvement survived a mean of 46.3 months (SE 6.9, 95\% Cl 32.7-59.9), compared to 31.5 months (SE 5.1, 95\% CI 28.0-45.0) in those who had cervical lymphadenopathy.

Twenty-two patients, referred by their GPs with a suspicion of head and neck cancer, were seen within two weeks (average 10 days) in a rapid access specialised clinic, as recommended by current national guidelines [11]. The mean duration from first clinic appointment to operation was 43.7 days (SE 37.1). The remaining patients were identified in routine ENT clinics or after referral from other nonspecialised hospital clinicians, with the average duration from referral to ENT clinic appointment of 21.4 days (SE 12.4 , range $2-46$ ) and the average duration from clinic appointment to operation of 53 days (SE 34.5). No statistical difference in survival probability was noted between these 
two groups $(P=0.37)$, although patients who had been seen within two weeks had better survival.

\section{Discussion}

\section{Synopsis of key findings}

The overall survival rate was $36.6 \%$, with a mean survival period of 42.4 months after surgery. Patients with primary laryngeal cancers had better survival probabilities than those with hypopharyngeal cancers. Those who had salvage surgery for laryngeal recurrence had significantly better survival probability than hypopharyngeal recurrence. Advanced nodal and $T$ stage were both poor prognostic indicators for survival. There was a non-significant survival advantage in patients who had primary surgery compared with those who had salvage surgery for recurrence after RT. Interestingly, it was observed that patients who had salvage surgery for laryngeal SCCa had better survival than those who underwent TL as the primary treatment modality, although this trend was not statistically significant.

\section{Comparisons with other studies}

Patients with hypopharyngeal cancer had poorer survival outcome than those with laryngeal cancer, comparable with results reported by Hall et al. [3]. Likewise, patients with recurrent laryngeal cancer had longer mean survival period than those with recurrent hypopharyngeal cancer [4]. Stoekli et al. reported that the 5-year DSS for 39 cases after salvage TL was 0.63 , with mortality rate of $49 \%$. In contrast, the overall mortality after salvage surgery in this study was $65 \%$. Other studies have reported poorer mortality rates, as high as $85 \%$ at 25 months follow-up in 20 salvage cases reported by Young et al. [5]. The present overall 5-year DSS was poorer than some reported studies. In a cohort of 83 Stage IV laryngeal SCCa studied by Spector et al., the 5-year DSS was higher at 0.45 , although the reported DFS of 0.29 was lower than this study [6]. The overall complication rate of $38 \%$ was lower than that reported by Hall et al. (48\%) [3]. No flap failure was reported in this study.

\section{Pharyngo-cutaneous fistula formation}

The reported incidence of PCF formation after laryngectomy varies from $6.5 \%$ to $20 \%$ in larger case series $[3,5,7,8]$. Only a small proportion of patients $(4 \%)$ in this study suffered with a PCF. This may be due to the higher rate of primary TL (75\%) performed compared to salvage laryngectomy. It is also our department's practice to commence early enteral feeding via the primary tracheo-oesophageal fistula rather than naso-gastric tube until contrast swallow confirms anastomotic integrity. Wakisaka et al. encountered a $27 \%$ rate of PCF after TL in their cohort of 63 cases [9]. They reported an increased incidence of PCF formation in patients who had prior RT or combination chemo-RT, and concluded that the addition of chemotherapy to irradiation delays PCF closure. In addition, Boscolo-Rizzo et al. reported that diabetes mellitus, preoperative hypoalbuminemia, chronic pulmonary diseases and chronic hepatopathy were independent predictors for PCF formation [10]. The low number of cases in this study precluded multivariate analysis for correlation of risk factors to occurrence of PCF.

\section{2-week wait ( $2 \mathrm{WW}$ ) rule for suspected head and neck cancer}

Patients referred under the $2 \mathrm{WW}$ rule had better survival outcome than those referred conventionally, although the difference was not statistically significant. Whilst this may demonstrate the benefits of rapid referral, earlier diagnosis and surgery, other factors also play a part including discussion in a formal MDT and introduction of national guidelines for the management of head and neck cancer [11]. The management of all patients in the current series were discussed in a MDT. Furthermore, the overall waiting time for ENT clinic appointment in our institution has been reducing, in keeping with current guidelines. The average period from date of referral to surgery differed only by 19 days between the two groups and this may also explain the statistical outcome. A 3-year review of over 1,1002WW referrals in a larger cancer centre reported that only $21.4 \%$ of these referrals were positive for head and neck cancer [12]. When compared with routine referral routes, Mckie et al. reported that the $2 \mathrm{WW}$ did not identify more early stage cancers [12]. Lyons et al. also found that $71 \%$ of patients diagnosed with cancer in their department were not referred by the fast track 2 WW system and that only $15 \%$ of patients who were referred to the fast track system were subsequently found to have cancer [13]. A structured search of PubMed revealed that most published studies to date were audits of compliance with the $2 \mathrm{WW}$ rule [14-16].

\section{Clinical applicability of the study}

To date, few UK centres have published outcomes following TL. Bajaj et al. reviewed outcomes of 59 patients over a 6-year period and reported that 5-year survival was $65.2 \%$ [17]. The patient cohort also varied from T1 to T4, which was similar to the present study. The results in this study have focused specifically on survival rates, complications and causes of death. Quality of life issues were not evaluated nor were mortality correlated with pre-existing medical comorbidities. Survival outcomes in patients referred under the 2WW for suspicious cancer have also been reported in this study. Similarities in survival outcomes with other studies do not negate the need for U.K. specific data as patient cohorts differ between these studies. Outcome data specific to the population being treated would improve our ability to council patients regarding important therapeutic decisions and end-of-life issues.

Selection criteria for TL have remained the same throughout the review period. In general, patients who had failed RT or those with T3 tumours of the larynx or hypopharynx were eligible for surgery. Transoral laser resection was introduced to the department in 2003 and some patients had TL after failed TOLR. The treatment protocol for RT for primary carcinoma was unchanged throughout the period of the study. Patients who had pre-TL tracheotomy, T4 tumours, neck node involvement ( $>1$ node, $>2 \mathrm{~cm}$, extracapsular spread) had post-TL RT. 
The results of this study represent the cumulative experience of TL of the department over a period of 14 years. The study cohort comprised of four groups: laryngeal or hypopharyngeal, primary treatment or salvage. It can be argued that to make better sense of the outcome, it would be better to consider the four groups individually. However, the number of patients in each group was relatively small. Furthermore, the aim of this retrospective review was to evaluate the clinical outcomes (survival rate, complications, PCF) following $\mathrm{TL}$, rather than tumour staging, primary site or outcomes based on specific treatment modality.

\section{Conclusions}

While there is an increasing tendency towards laryngeal conservation, TL (primary or salvage) remains a robust treatment option in selected patients. This treatment modality will continue to form part of the armamentarium of treatment options available to patients treated in our institution. The results of this study represent the clinical outcome of a cohort of patients after TL from the perspective of a district general hospital in the United Kingdom. Unlike previous publications which have focused on outcomes based on tumour staging, we have presented survival probabilities following TL. Outcome data such as this would improve our ability to council patients regarding important therapeutic decisions and end-of-life issues. Future reports from this department would examine the correlation of medical co-morbidities (ACE-27), alcohol and smoking with survival probabilities.

\section{Disclosure of interest}

The authors declare that they have no conflicts of interest concerning this article.

\section{References}

[1] Birchall M, Pope L. Tumours of the larynx. In: Gleeson M, Scott-Brown $M$, editors. Otorhinolaryngology. London: Arnold Hodder; 2008. p. 2598-9.
[2] Cancer Research UK. Disponible à: http://info.cancerresearchuk.org/cancerstats/types/larynx/?a=5441. Évalué le: 29 décembre 2008.

[3] Hall FT, O'Brien CJ, Clifford AR, et al. Clinical outcome following total laryngectomy for cancer. ANZ J Surg 2003;73:300-5.

[4] Stoeckli SJ, Pawlik AB, Lipp M, et al. Salvage surgery after failure of nonsurgical therapy for carcinoma of the larynx and hypopharynx. Arch Otolaryngol Head Neck Surg 2003;126:1473-7.

[5] Young VN, Mangus BD, Bumpous JM. Salvage laryngectomy for failed conservative treatment of laryngeal cancer. Laryngoscope 2008;118:1561-8.

[6] Spector GJ, Sessions DG, Lenox J, et al. Management of Stage IV glottic carcinoma: therapeutic outcomes. Laryngoscope 2004;114:1438-46.

[7] Theile DR, Robinson DW, Theile DE, et al. Free jejunal interposition reconstruction after pharyngolaryngectomy: 201 consecutive cases. Head Neck 1995;17:83-8.

[8] Soylu L, Kiroglu M, Aydogan B, et al. Pharyngocutaneous fistula following laryngectomy. Head Neck 1998;20:22-5.

[9] Wakisaka N, Murono S, Kondo S, et al. Post-operative pharyngocutaneous fistula after laryngectomy. Auris Nasus Larynx 2008;35:203-8.

[10] Boscolo-Rizzo P, De Cillis G, Marchiori C, et al. Multivariate analysis of risk factors for pharyngocutaneous fistula after total laryngectomy. Eur Arch Otorhinolaryngol 2008;265:929-36.

[11] Department of Health. London, U.K. Referral guidelines for suspected cancer, 2000. p. 39-41.

[12] McKie C, Ahmad UA, Fellows S, et al. The 2-week rule for suspected head and neck cancer in the United Kingdom: referral patterns, diagnostic efficacy of the guidelines and compliance. Oral Oncol 2008;44:851-6.

[13] Lyons M, Philpott J, Hore I, et al. Audit of referrals for head and neck cancer - the effect of the 2-week, fast track referral system. Clin Otolaryngol Allied Sci 2004;29:143-5.

[14] Webb CJ, Benton J, Tandon S, et al. Head and neck cancer waiting times. Clin Otolaryngol 2007;32:293-6.

[15] Hobson JC, Malla JV, Sinha J, et al. Outcomes for patients referred urgently with suspected head and neck cancer. J Laryngol Otol 2008;122:1241-4.

[16] Duvvi SK, Thomas L, Vijayanand S, et al. Two-week rule for suspected head and neck cancer. A study of compliance and effectiveness. J Eval Clin Pract 2006;12:591-4.

[17] Bajaj Y, Shayah A, Sethi N, et al. Clinical outcomes of total laryngectomy for laryngeal carcinoma. Kathmandu Univ Med J (KUMJ) 2009;7:258-62. 